Pacific Journal of Mathematics

ON THE CLASSIFICATION OF LINDENSTRAUSS SPACES 


\section{ON THE CLASSIFICATION OF LINDENSTRAUSS SPACES}

\section{H. Elton Lacey}

A Lindenstrauss space is a real Banach space $X$ such that its dual $X^{*}$ is linearly isometric to $L_{1}(\mu)$ for some measure $\mu$. The purpose of this paper is to describe how certain classical types of Lindenstrauss spaces are characterized by mappings from a compact Hausdorff space $S$ into $C(S)^{*}$.

Let $S$ be a compact Hausdorff space and $\rho: S \rightarrow C(S)^{*}$ a bounded function such that for each $f \in C(S)$, the function $f_{\rho}$ defined by $f_{\rho}(s)=\int f d \rho(s)$ is integrable with respect to each regular Borel measure on $S$. Thus $\rho$ induces a natural bounded linear operator $P$ on $C(S)^{*}$ defined by $(P \mu)(f)=\int f_{\rho} d \mu$ for all $\mu \in C(S)^{*}$ and $f \in C(S)$. If (i) $\|\rho(s)\| \leqq 1$ for all $s \in S$, and (ii) whenever $\mu \in C(S)$ and $\int f d \mu=0$ for all $f \in C(S)$ with $f=f_{\rho}$, then $\int f_{\rho} d \mu=0$ for all $f \in C(S)$, then $\rho$ is said to be an affine mapping.

It was shown in [1] that if $\rho$ is affine, then $X_{\rho}=\left\{f \in C(S): f=f_{\rho}\right\}$ is a Lindenstrauss space, $P$ is a contractive projection on $C(S)^{*}$ with kernel equal to $X_{\rho}^{\perp}=\left\{\mu \in C(S)^{*}: \mu(f)=0\right.$ for all $\left.f \in X_{\rho}\right\}$. Moreover, the restriction mapping $\mu \rightarrow \mu \mid X_{\rho}$ is a linear isometry from the range of $P$ onto $X_{\rho}^{*}$.

Condition (ii) of the definition of an affine mapping is usually the hardest to verify. In [7] Gleit gives a nontrivial example when $\rho(s) \geqq 0$ for all $s \in S$. Although he did not actually use this terminology, careful inspection of his proof yields that the mapping he postulates is indeed an affine mapping. In fact, slight modifications in his proof yields the following general result.

THEOREM 1. (Gleit). Let $S$ be a compact Hausdorff space and $T$ a closed subset of $S$. Let $\rho: S \rightarrow C(S)^{*}$ be a mapping such that (a) $\|\rho(s)\| \leqq 1$ for all $s \in S$,

(b) $\rho(s)=\varepsilon_{s}$ (i.e., point evaluation) for $s \in S \backslash T$,

(c) for $T_{2}=\left\{s \in T: \rho(s)=\varepsilon_{s}\right\}$ and $T_{1}=T \backslash T_{2}$, we suppose that $T_{1} \neq S$ and $|\rho(s)|\left(T_{1}\right)=0$ for all $s \in T_{1}$,

(d) $\rho \mid T$ is weak* continuous.

Then $\rho$ is an affine mapping and $X_{\rho}^{*}$ is, in fact, linearly isometric to $\left\{\mu \in C(S)^{*}:|\mu|\left(T_{1}\right)=0\right\}$.

As mentioned above, Gleit assumes in addition that $\rho(s) \geqq 0$ for 
all $s \in S$. This assumption yields that $X_{\rho}$ is ordered and $X_{\rho}^{*}$ is an $L$ space in its dual ordering. This will be proved in general in Theorem 2. For this and other results certain classical types of Lindenstrauss spaces are recalled.

$C(S)$ : the Banach space of all continuous real valued functions on a compact Hausdorff space $S$.

$C_{0}(L S)$ : the Banach space of all continuous real valued functions which vanish at infinity on a locally compact Hausdorff space $L S$.

$A(K)$ : the Banach space of all affine continuous functions on a compact Choquet simplex $K$.

$A_{0}(K)$ : the Banach space of all affine continuous functions on a compact Choquet simplex space $K$ which vanish at a fixed extreme point of $K$.

$C_{o}(S)$ : the set of all $f \in C(S)$ such that $f(s)=-f(\sigma s)$ for all $s \in S$, where $\sigma$ is a homeomorphism on $S$ such that $\sigma^{2}$ is the identity.

$M$ space: the set of all $f \in C(S)$ satisfying a fixed set of relations $\left\{s_{i}, s_{i}^{\prime}, \lambda_{i}\right\}$ where $s_{i}, s_{i}^{\prime} \in S, \lambda_{i} \in[0,1]$ and $f$ satisfies $f\left(s_{i}\right)=\lambda_{i}\left(s_{i}^{\prime}\right)$ for all $i$ in some index set.

$G$ space: the set of all $f \in C(S)$ satisfying a fixed set of relations $\left\{s_{i}, s_{i}^{\prime}, \lambda_{i}\right\}$ where $s_{i}, s_{i}^{\prime}$ are in $S, \lambda_{i} \in[-1,1]$ and $f$ satisfies $f\left(s_{i}\right)=\lambda_{i} f\left(s_{i}^{\prime}\right)$ for all $i$ in some index set.

THEOREM 2. Let $S$ be a compact Hausdorff space and $\rho: S \rightarrow$ $C(S)^{*}$ be an affine mapping. If $\rho(s) \geqq 0$ for all $s \in S$, then $X_{\rho}$ is an $A_{0}(K)$ space. If $\rho(s) \geqq 0$ and $\|\rho(s)\|=1$ for all $s \in S$, then $X_{\rho}$ is an $A(K)$ space. Conversely, each $A_{0}(K)$ and $A(K)$ space can be so represented.

Proof. Let $P$ be the projection determined by $\rho$. Let $r$ be the restriction mapping from the range $N$ of $P$ onto $X^{*}$. Then $r$ is a linear isometry and is clearly positive from $N$ to $X_{\rho}^{*}$ where $X_{\rho}^{*}$ has the dual ordering from the induced ordering on $X_{\rho}$ from $C(S)$.

Now $N$ is a sublattice of $C(S)^{*}$. For, if $\mu, \nu$ are in $N$, then since $P$ is positive, $P(\mu \vee \nu) \geqq \mu \vee \nu$ and since $P$ is contractive, $\|\mu \vee \nu\| \geqq\|P(\mu \vee \nu)\|$. Thus it follows that $\mu \vee \nu=P(\mu \vee \nu)$. Hence it remains only to show that $r$ is order preserving to establish that $X_{\rho}$ is an $A_{0}(K)$ space (see [2]). Let $x^{*} \in X_{\rho}^{*}$ be positive and have norm one and let $W=\left\{y^{*} \in X^{*}: 0 \leqq y^{*},\left\|y^{*}\right\| \leqq 1\right\}$. Clearly $W$ is a weak* closed convex set in $X_{\rho}^{*}$. Let $S_{0}$ be the weak* closure of the extreme points of $W$. Since each nonzero extreme point of $W$ is the image (under restriction) of an extreme point of the unit sphere of $C(S)^{*}$ and since the positive cone of $X_{\rho}$ is nonzero, it follows that $S_{0}$ is contained in the image of $\left\{\varepsilon_{s}: s \in S\right\} \cup\{0\}=S_{1}$ 
under restriction. Now, there is a probability measure $\mu$ on $S_{0}$ such that $\int y^{*}(f) d \mu\left(y^{*}\right)=x^{*}(f)$ for all $\mathrm{f} \in X_{\rho}$ (see [19]) and by [8] there is a probability measure $\nu$ on $S_{1}$ such that $\int \hat{f} d \nu=\int y^{*}(f) d \mu\left(y^{*}\right)=x^{*}(f)$ for all $f \in X_{\rho}$, where $\hat{f}$ is defined by $\hat{f}\left(\varepsilon_{s}\right)=f(s)$ and $\hat{f}(0)=0$. Thus $\nu^{\prime}$ defined by $\nu^{\prime}(A)=\nu\left(\left\{\varepsilon_{s}: s \in A\right\}\right)$ is a nonnegative measure on $S$ and $\int f d \nu^{\prime}=\int \hat{f} d \nu$ for all $f \in X_{\rho}$ (since $\hat{f}(0)=0$ ). If $r P \nu^{\prime}=x^{*}$, then since $P \nu^{\prime} \geqq 0$, the proof is complete. But, $\int f d \nu^{\prime}=\int \hat{f} d \nu=\int y^{*}(f) d \mu\left(y^{*}\right)=$ $x^{*}(f)$ for $f \in X_{\rho}$.

If, in addition, $\|\rho(s)\|=1$ for all $s \in S$, then $1 \in X_{\rho}$ and it follows that $X_{\rho}$ has a strong order unit and so $X_{\rho}$ is an $A(K)$ space where $K$ is a compact Choquet simplex.

Conversely, if we are given a simplex space $A_{0}(K)$, then without loss of generality $K=\left\{x^{*} \in A_{0}(K)^{*}: 0 \leqq x^{*},\left\|x^{*}\right\| \leqq 1\right\}$. For each $x^{*} \in K$, let $\mu_{x^{*}}$ denote the unique maximal probability measure representing $x^{*}$ (see [21]). It is well known that if $S$ is equal to the weak* closure of the extreme points of $K$ and $\rho\left(x^{*}\right)$ is the unique maximal probability measure on $S$ representing $x^{*}, \rho$ is an affine mapping and $A(K)=X_{\rho}$. Let $P$ be the projection associated with $\rho$. Then it is also well known that kernel $P=A(K)^{\perp}$ (the reader can see [1] for a proof of this).

Now, let $\tilde{\rho}\left(x^{*}\right)=\rho\left(x^{*}\right)$ for $x^{*} \neq 0$ and $\tilde{\rho}(0)=0$. Then $\tilde{\rho}$ is an affine mapping on $S$ and the associated projection $\widetilde{P}$ is given by $\widetilde{P} \mu=\mu-\mu(0) \cdot \varepsilon_{0}$. From this it follows that the kernel of $\widetilde{P}$ is equal to $\left\{\mu:\left(\mu-\mu(0) \cdot \varepsilon_{0}\right) \in A(K)^{\perp}\right\}=\left\{\mu: \mu \in A_{0}(K)^{\perp}\right\}$. Thus it follows that $X_{\tilde{\rho}}=A_{0}(K)$.

Let $X$ be a Banach space, $V$ the unit sphere of $X^{*}, S$ the weak* closure of the set of extreme points of $V$, and $\sigma: V \rightarrow V$ be defined by $\sigma\left(x^{*}\right)=-x^{*}$ for all $x^{*} \in V$. The following theorem was also proved in [1]. The notation is as above.

Theorem 3. Let $X$ be a Lindenstrauss space. Then $\rho: S \rightarrow C(S)^{*}$ defined by $\rho\left(x^{*}\right)=(1 / 2)[\mu-\mu \circ \sigma]$, where $\mu$ is a maximal probability measure on $V$ (supported on $S$ ) representing $x^{*}$, is an affine mapping and $X=X_{\rho}$.

Theorem 3 will be used to characterize some of the types of Lindenstrauss spaces in terms of affine mappings.

THEOREM 4. Let $S$ be a compact Hausdorff space and $\rho: S \rightarrow C(S)^{*}$ be a weak* continuous affine mapping. Then $X_{\rho}$ is a $C_{\sigma}\left(S_{0}\right)$ space for some compact Hausdorff space $S_{0}$. Conversely, any $C_{\sigma}\left(S_{0}\right)$ space can be so represented. 
Proof. Let $\mu \in C(S)^{*}$. Then $\int f d \mu=\int f d P \mu=\int f_{\rho} d \mu$ for all $f \in X_{\rho}$. Thus $\int f_{\rho} d \mu=\int f_{\rho} d P \mu=\int f_{\rho \rho} d \mu$ for all $f \in C(S)$ Since $\rho$ is weak* continuous, $f_{\rho}$ and $f_{\rho \rho}$ are in $C(S)$. Thus $f_{\rho}=f_{\rho \rho}$ for all $f \in C(S)$ and $X_{\rho}=\left\{f_{\rho}: f \in C(S)\right\}$. Moreover, the operator $Q$ defined by $Q(f)=$ $f_{\rho}$ for $f \in C(S)$ is a constructive projection of $C(S)$ onto $X_{\rho}$. Consequently $X_{o}$ is a $C_{\sigma}\left(S_{0}\right)$ space for some compact Hausdorff space $S_{0}$ by [1].

On the other hand, if $X=C_{\sigma}\left(S_{0}\right)$ for some compact Hausdorff space $S_{0}$, then the weak* closure, $S$, of the set $E$ of extreme points of the unit sphere of $X^{*}$ is either $E$ or $E \cup\{0\}$ depending on whether or not $\sigma$ is fixed point free (see [16]). In particular for $s \in S_{0}$ and $\sigma(s) \neq s, \quad\left(\varepsilon_{s} \mid X\right) \in E$. Thus by Theorem 3 the mapping defined by $\rho(s)=(1 / 2)\left(\varepsilon_{s}-\varepsilon_{\sigma(s)}\right)$ defines an affine mapping and $X=X_{\rho}$. Clearly $\rho$ is weak* continuous.

From the definition of an $M$ space, if $\rho: S \rightarrow C(S)^{*}$ is any function which takes its values in $[0,1] S=\left\{\lambda \varepsilon_{s}: 0 \leqq \lambda \leqq 1, s \in S\right\}$, then $X_{o}=\left\{f \in C(S): f(s)=\int f d \rho(s)\right.$ for all $\left.s \in S\right\}$ is an $M$ space.

Theorem 5. Let $X$ be an $M$ space. Then there is a compact Hausdorff space $S$ and an affine mapping $p: S \rightarrow C(S)^{*}$ such that $\rho$ takes its values in $[0,1] S$ and $X=X_{\rho}$.

Proof. Let $S$ be the weak* closure of the set $E$ of positive extreme points of the unit sphere of $X^{*}$. It is well known that each element $x^{*} \in S$ is of the form $\lambda y^{*}$ with $y^{*} \in E$ and $0 \leqq \lambda \leqq 1$ [9]. Moreover this representation is unique if $x^{*} \neq 0$. Thus $\rho\left(x^{*}\right)=$ $\lambda \varepsilon_{y^{*}}$ defines an affine mapping and $X_{o}=X$ (see the proof of Theorem 2).

Similarly, if $\rho: S \rightarrow C(S)^{*}$ takes its values in $[-1,1] S=\left\{\lambda \varepsilon_{s}:-1 \leqq\right.$ $\lambda \leqq 1, s \in S\}$, then $X_{\rho}=\left\{f \in C(S): f(s)=\int f d \rho(s)\right.$ for all $\left.s \in S\right\}$ in a $G$ space. On the other hand, if $X$ is a $G$ space and $S$ is the weak* closure of the set $E$ of extreme points of the unit sphere of $X^{*}$, then Fakhoury has shown in [3] that $S \subset[-1,1] E$. Thus for any $x^{*} \in S, x^{*}=\alpha y^{*}+(1-a)\left(-y^{*}\right)$ for some $0 \leqq \alpha \leqq 1$ and $y^{*} \in E$. Moreover, $\mu=\alpha \varepsilon_{y^{*}}+(1-\alpha) \varepsilon_{-y^{*}}$ is a maximal measure representing $x^{*}$ and $(1 / 2)[\mu-\mu \circ \sigma]=(1 / 2)\left[(2 \alpha-1) \varepsilon_{y^{*}}+(1-2 \alpha) \varepsilon_{-y^{*}}\right]=\rho\left(x^{*}\right)$ defines an affine mapping with $X=X_{\rho}$ by Theorem 3 .

If $S$ is a compact Hausdorff space, then $\rho$ defined by $\rho(s)=\varepsilon_{s}$ is clearly an affine mapping and $C(S)=X_{\rho}$. On the other hand, if $L S$ is a locally compact noncompact Hausdorff space, then the weak* closure $S_{0}$ of the set $E$ of positive extreme points of the unit sphere of $C_{0}(L S)^{+}$is $E \cup\{0\}$. Clearly $S_{0}$ can be thought of as $S \cup\{0\}$ since 
$E$ is homeomorphic to $S$. Let $\rho(s)=\varepsilon_{s}$ for $s \in S$ and $\rho(0)=0$. Then as in Theorem 5, $\rho$ is an affine mapping and $X_{\rho}=C_{0}(L S)$.

THEOREM 6. Let $S$ be a compact Hausdorff space and $\rho: S \rightarrow$ $C(S)^{*}$ be a positive weak* continuous affine mapping. Then $X_{\rho}$ is a $C_{0}(L S)$ space.

Proof. By Theorems 2 and $4, X_{\rho}$ is simultaneously a $C_{\sigma}\left(S_{0}\right)$ space and an $A_{0}(K)$ space. Thus by [16] $X_{\rho}$ is a $C_{0}(L S)$ space for some locally compact Hausdorff space $L S$.

Open questions. The results in this paper are in the isometric theory of Banach spaces. It is natural to ask about the isomorphic theory. The isomorphic analogue to a Lindenstrauss space is a $\mathscr{L}_{\infty}$ space. A Banach space $X$ is said to be a $\mathscr{L}_{\infty, \lambda}$ space if for each finite dimensional subspace $Y$ of $X$ there is finite dimensional subspace $Z$ of $X$ with $Y \subset Z$ and $d\left(Z, l_{\infty}(\operatorname{dim} Z)\right)=\inf \left\{\|T\|\left\|T^{-1}\right\|: T: Z \rightarrow\right.$ $l_{\infty}(\operatorname{dim} Z)$ is $\left.1-1\right\} \leqq \lambda$.

Using proofs similar to those in [1] one can show that is $F$ is a compact Hausdorff space and $\rho: S \rightarrow C(S)^{*}$ has the properties of an affine mapping except that we only assume that $\sup \|\rho(s)\|<\infty$ instead of $\|\rho(s)\| \leqq 1$ for all $s \in S$, then $X_{\rho}$ is an $\mathscr{L}_{\infty}$ space.

Question 1. Is it possible to construct each $\mathscr{L}_{\infty}$ space from such a mapping?

Another question which is well known is the following.

Question 2. Is every $\mathscr{L}_{\infty}$ space isomorphic to a Lindenstrauss space? Recently, Benyamini and Lindenstrauss [21] have shown that there are separable Lindenstrauss spaces $X$ and $Y$ such that $X^{*}$ is separable and $Y^{*}$ is nonseparable and both $X$ and $Y$ are not isomorphic to a complemented subspace of a $C(S)$ space.

Some questions which arise in the isometric theory are discussed below. Let $S$ be a compact Hausdorff space and $X \subset C(S)$ a closed linear subspace containing 1 . Suppose further that $\left\{s \in S: \varepsilon_{s} \mid X\right.$ is an extreme point of the unit sphere of $\left.X^{*}\right\}=\partial_{X} S$ is dense in $X$. Then $X=A(K)$ where $K=\left\{x^{*} \in X^{*}: x^{*}(1)=1=\left\|x^{*}\right\|\right\}$ has the weak* topology and the extreme points of $K$ are homeomorphic to $\partial_{X} S$ and their closure is homeomorphic to $S$. In particular, each maximal measures on $K$ is supported on $S$.

Question 3. Is there an affine mapping $\rho$ of $S$ into the maximal measures on $K$ such that $\rho(s)=\varepsilon_{s}$ if and only if $s \in \partial_{X} S$ ? 
In [20] Rao has shown that there is a Borel measurable mapping when $S$ is metrizable metrizable.

This is related to the following question.

Question 4. If $S$ is a metrizable compact Hausdorff space and $T \subset S$ is a dense $G_{\delta}$ set, is there a compact Choquet simplex with extreme points homeomorphic to $T$ and their closure homeomorphic to $S$ ?

Let $X \subset C(S)$ be as above. It is shown in [6] that if $X$ is a Lindenstrauss space, then $X$ is maximal with respect to $\partial_{X} S$. That is, if $X \subset Y$ and $\partial_{Y} S=\partial_{X} S$, then $X=Y$. An easy application of Zorn's lemma shows that for any such $X \subset C(S)$ there is a maximal $Y \supset X$ with respect to $\partial_{X} S$.

Question 5. Is any $X$ maximal with respect to $\partial_{X} S$ a Lindenstrauss space?

\section{REFERENCES}

1. J. B. Bednar and H. E. Lacey, Concerning Banach spaces whose duals are L spaces, Pacific J. Math., 41 (1972), 13-24.

2. E. B. Davies, On the Banach space duals of certain spaces with the Riesz decomposition property, Quart. J. Math., Oxford 18 (1967), 109-111.

3. H. Fakhoury, Preduax de $L$ espaces; Properties du $G$ espaces et des $C$ espaces,

C. R. Acad. Sci. Paris t 271 (1970), Series A, 941-944.

4. - Sélections Linéaires Associees au Théoréme de Hahn-Banach, (preprint).

5 . — - Une Characterisation des $L$ espaces duax, (to appear).

6. D. A. Edwards and G. Vincent-Smith, A Weierstrass Stone theorem for Choquet simplexes, Ann. Inst. Fourier Grenoble 18 (1968), 261-282.

7. A. Gleit, On the existence of simplex spaces, Israel J. Math., 9 (1971), 199-209.

8. J. Hardy and H. E. Lacey, Extensions of regular Borel measures, Pacific J. Math., 24 (1968), 277-282.

9. S. Kakutani, Concrete representations of abstract $M$ spaces, Ann. Math., 42 (1941), 994-1024.

10. H. E. Lacey, $A$ note concerning $A^{*}=L_{1}(\mu)$, Proc. Amer. Math. Soc., 29 (1971), $525-528$.

11. H. E. Lacey, and P. D. Morris, Continuous linear operators on spaces of continuous functions, Proc, Amer. Math. Soc., 17 (1966), 848-853.

12. - On spaces of type $A(K)$ and their duals, Proc. Amer. Math. Soc., 23 (1969), 151-157.

13. A. J. Lazar, The unit ball in conjugate $L_{1}$ spaces, Duke Math. J., 39 (1972), 1-8.

14. J. Lindenstrauss and A. Pelczynski, Absolutely summing operators in $L_{p}$ spaces and their applications, Studia Math., 29 (1968), 275-326.

15. J. Lindenstrauss and H. P. Rosenthal, The $\mathscr{L}_{p}$ spaces, Israel J. Math., 7 (1969), 325-349.

16. J. Lindenstrauss and D. E. Wulbert, On the classification of the Banach spaces whose duals are $L_{1}$ spaces, J. Functional Anal., (1969), 332-349.

17. A. Pelczynski, On Banach spaces containing $L_{1}(\mu)$, Studia Math., 30 (1968), 231246. 
18. A. Pelczynski and Z. Semaderi, Spaces of continuous functions III, Studia Math., 18 (1959), 211-222.

19. R. R. Phelps, Lectures on Choquet's Theorem, Math. Studies, D. Van Nostrand, Princeton, 1966.

20. M. M. Rao, Borel Selections of Representing Measures, Aarus University notes, 1970.

21. Y. Benyamini and J. Lindenstrauss, A predual of $l_{1}$ which is not isomorphic to a $C(K)$ spaces, (preprint).

Received December 14, 1971 and in revised form January 18, 1973.

UNIVERSTTY OF TEXAS AT AUSTIN 



\section{PACIFIC JOURNAL OF MATHEMATICS}

\section{EDITORS}

D. Gilbarg and J. Milgram

Stanford University

Stanford, California 94305

R. A. Beaumont

University of Washington

Seattle, Washington 98105
J. DUGUNDJI* Department of Mathematics

University of Southern California Los Angeles, California 90007

RICHARD ARENS

University of California

Los Angeles, California 90024

\section{ASSOCIATE EDITORS}

E. F. BeCKenbach

B. H. NeUMaNN

F. WOLF

K. Yoshida

\section{SUPPORTING INSTITUTIONS}

UNIVERSITY OF BRITISH COLUMBIA

UNIVERSITY OF SOUTHERN CALIFORNIA

CALIFORNIA INSTITUTE OF TECHNOLOGY

UNIVERSITY OF CALIFORNIA

MONTANA STATE UNIVERSITY

STANFORD UNIVERSITY

UNIVERSITY OF TOKYO

UNIVERSITY OF NEVADA

UNIVERSITY OF UTAH

NEW MEXICO STATE UNIVERSITY

WASHINGTON STATE UNIVERSITY

OREGON STATE UNIVERSITY

UNIVERSITY OF OREGON

OSAKA UNIVERSITY

UNIVERSITY OF WASHINGTON

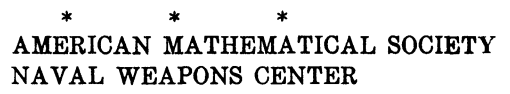

* C. DePrima will replace J. Dugundji until August 1974. 


\section{Pacific Journal of Mathematics}

\section{Vol. 47, No. $1 \quad$ January, 1973}

K. Adachi, Masuo Suzuki and M. Yoshida, Continuation of holomorphic

mappings, with values in a complex Lie group ....................

Michael Aschbacher, A characterization of the unitary and symplectic groups

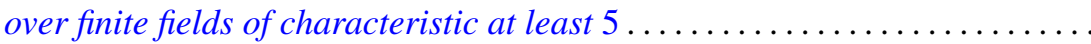

Larry Eugene Bobisud and James Calvert, Energy bounds and virial theorems for abstract wave equations....................................

Christer Borell, A note on an inequality for rearrangements ................

Peter Southcott Bullen and S. N. Mukhopadhyay, Peano derivatives and general

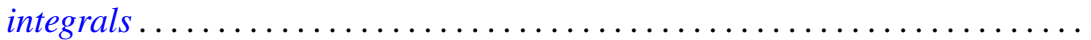

Wendell Dan Curtis, Yu-Lee Lee and Forrest Miller, A class of infinite dimensional subgroups of $\operatorname{Diff}^{r}(X)$ which are Banach Lie groups .........

Paul C. Eklof, The structure of ultraproducts of abelian groups ...............

William Alan Feldman, Axioms of countability and the algebra $C(X) \ldots \ldots \ldots$

Jack Tilden Goodykoontz, Jr., Aposyndetic properties of hyperspaces...........

George Grätzer and J. Płonka, On the number of polynomials of an idempotent algebra. II ...........................................

Alan Trinler Huckleberry, The weak envelope of holomorphy for algebras of

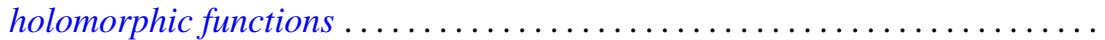

John Joseph Hutchinson and Julius Martin Zelmanowitz, Subdirect sum decompositions of endomorphism rings . . . . . . . . . . . . . . . .

Gary Douglas Jones, An asymptotic property of solutions of

$y^{\prime \prime \prime}+p y^{\prime}+q y=0$.

Howard E. Lacey, On the classification of Lindenstrauss spaces .

Charles Dwight Lahr, Approximate identities for convolution measure algebras.

George William Luna, Subdifferentials of convex functions on Banach

spaces.

Nelson Groh Markley, Locally circular minimal sets. .

Robert Wilmer Miller, Endomorphism rings of finitely generated projective modules

Donald Steven Passman, On the semisimplicity of group rings of linear

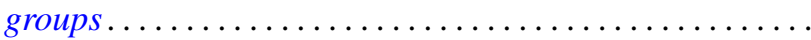

Bennie Jake Pearson, Dendritic compactifications of certain dendritic spaces.

Ryōtarō Satō, Abel-ergodic theorems for subsequences ...... .

Henry S. Sharp, Jr., Locally complete graphs. . .

Harris Samuel Shultz, A very weak topology for the Mikusinski field of

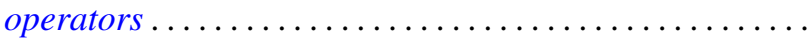

Elena Stroescu, Isometric dilations of contractions on Banach spaces ...

Charles W. Trigg, Versum sequences in the binary system ... . .

William L. Voxman, On the countable union of cellular decompositions of n-manifolds 